\title{
Dietary isoflavone intake is associated with a reduced risk of myelodysplastic syndromes
}

\author{
Ping Liu ${ }^{1}$, C. D’Arcy J. Holman ${ }^{1}$, Jie Jin ${ }^{2}$ and Min Zhang ${ }^{1,3 *}$ \\ ${ }^{1}$ School of Population Health, The University of Western Australia, 35 Stirling Highway, Crawley, Perth, WA 6009, Australia \\ ${ }^{2}$ Department of Haematology, The First Affiliated Hospital, Zhejiang University College of Medicine, 79 Qingchun Road, \\ Hangzhou, Zhejiang 310003, People's Republic of China \\ ${ }^{3}$ Centre for Healthcare Resilience and Implementation Science, Australian Institute of Health Innovation, Macquarie \\ University, Level 6, 75 Talavera Road, NSW 2109, Australia
}

(Submitted 6 May 2015 - Final revision received 27 July 2015 - Accepted 25 August 2015 - First published online 13 October 2015 )

\section{Abstract}

Isoflavones have been suggested to have protective effects on certain cancers. However, the association of soya foods or dietary isoflavones with the risk of myelodysplastic syndromes (MDS) has not been examined. Thus, the aim of this hospital-based case-control study undertaken in China in 2012-2013 was to investigate the association between dietary isoflavone intake and MDS risk. The analysis included 208 cases aged 19-85 years with MDS and 208 controls individually matched to the cases by sex, birth quinquennium and residential locality. Information on habitual food intakes, including nine items of soya foods, was sought from in-person interviews using a validated 107-item FFQ. Dietary intakes of daidzein, genistein, glycitein and total isoflavones were estimated using the 2008 US Department of Agriculture Isoflavone Database. OR were calculated from conditional logistic regression after adjustment for potential confounding by demographics, lifestyle and dietary factors. The mean daily intake of total isoflavones was $19.0 \mathrm{mg}$ in cases and $23.0 \mathrm{mg}$ in controls. Dietary intake of isoflavones was inversely associated with the risk of MDS. The adjusted OR in the highest tertile compared with the lowest tertile of intake were 0.43 (95\% CI 0.21, 0.85) for daidzein, 0.36 (95\% CI 0.18, 0.74) for genistein, 0.49 (95\% CI 0.25, 0.97) for glycitein and 0.40 (95\% CI 0.20, $0 \cdot 81$ ) for total isoflavones. The findings suggest that higher dietary intake of isoflavones is associated with a reduced risk of MDS in a Chinese population.

\section{Key words: Isoflavones: Soya foods: Myelodysplastic syndromes: Case-control studies}

Myelodysplastic syndromes (MDS) are a complex and heterogeneous group of clonal haematopoietic stem cell malignancies, characterised by dysplastic and ineffective haematopoiesis, peripheral cytopenia(s) and an elevated risk of progression into acute myeloid leukaemia ${ }^{(1)}$. The average annual age-adjusted incidence rate is lower in Asians (1-2/100 000 person-years $(\mathrm{PY}))^{(2,3)}$ than that in Western populations $(3-4 / 100000 \mathrm{PY})^{(4,5)}$. The variation in incidence rates across populations may partly be attributed to the differences in dietary factors.

Compared with Western diets, traditional diets in Asians customarily consist of more foods from plants, particularly soya foods $^{(6)}$. The lower risk of MDS in Asians might suggest that some components from soya foods may have potential chemopreventive properties. Of the bioactive components, isoflavones are naturally occurring compounds in plants and are almost exclusively present in soya foods. Genistein and daidzein are the two major isoflavones, and glycitein is the minor one, accounting for approximately 50, 40 and $10 \%$, respectively, of the total soyabean isoflavone content ${ }^{(7)}$. Although a protective role of isoflavones has been observed for certain cancers, such as breast, prostate and gastrointestinal cancers ${ }^{(6,8)}$, none of the previous studies to date has reported the associations of soya foods or dietary isoflavone intake with the risk of MDS. We presented the data from a case-control study herein to investigate the association between dietary isoflavone intake and MDS risk in a Chinese population whose diet is rich in soya.

\section{Methods}

\section{Study design and participants}

A hospital-based case-control study was conducted in Hangzhou, the capital city of Zhejiang province in southeast China, between September 2012 and December 2013. Adult de novo MDS patients were identified from the medical records of the Department of Haematology at The First Affiliated Hospital of

\footnotetext{
Abbreviation: MDS, myelodysplastic syndromes.
}

* Corresponding author: Dr M. Zhang, fax +6128088 6234, email min.zhang@mq.edu.au 
Zhejiang University. Details of the study design and participants' recruitment have been described elsewhere ${ }^{(9)}$. In brief, male and female patients were eligible as cases if they met the 2008 WHO MDS diagnostic criteria, and if they were $\geq 18$ years old. Patients were excluded if they had secondary MDS or additional malignancy. Moreover, potential cases were excluded if there was the possibility of peripheral blood cytopenias associated with nutritional deficiencies, inflammation or infection, aplastic anaemia, acute myeloid leukaemia, myelodysplasticmyeloproliferative neoplasms or myeloproliferative neoplasms ${ }^{(1)}$. All relevant clinical and laboratory reports were reviewed daily. A total of 208 patients aged between 19 and 85 years were included as cases in the final analysis (response 95\%). For these cases, the median time interval between the date of diagnosis and the date of interview was $60 \mathrm{~d}$, and $79 \%$ of the cases were recruited within 1 year of diagnosis. Of the 208 cases, the major morphologic subtypes were refractory anaemia with excess of blasts $(41.4 \%)$ and refractory cytopenia with multilineage dysplasia (36.1\%). The minor subtypes were refractory cytopenia with unilineage dysplasia (12.5\%), MDS unclassifiable $(6.7 \%)$ and refractory anaemia with ring sideroblasts $(3 \cdot 4 \%)$.

At the same time as the cases were recruited, each control was selected as the first attendee to match with each case on sex, birth quinquennium and residential locality (urban or rural). Potential controls were excluded if they were not matched to their corresponding cases by these predefined matching factors, or if they had a previous diagnosis of MDS or another malignancy. The controls were recruited from the outpatient departments of the participating hospital (response $91 \%)$. Among the recruited controls, 107 (51\%) were members of the general population undergoing a routine health examination at the Medical Examination Centre; fifty-two (25\%) were outpatients from the ophthalmologic clinic; and forty-nine (24\%) were visitors who attended with family members or friends at the outpatient departments of the hospital. The study protocol was approved by the Human Research Ethics Committee of the University of Western Australia and the ethics committee of the participating hospital.

\section{Questionnaire and interview}

Participants were briefed regarding the general aims of the study to investigate dietary and lifestyle factors, confidentiality and anonymity issues. An appointment for an interview was made after obtaining their written informed consent via an initial contact. Face-to-face interviews were then conducted by the first author and a trained local research assistant. Each interview usually took 30-40 min. A structured questionnaire was used to elicit information on the following: (i) demographic characteristics and detailed lifestyle, such as education, weight and height, smoking status, tea and alcohol use, and physical activity; (ii) habitual dietary intake assessed by a quantitative FFQ; (iii) reproductive factors and family history of cancer; and (iv) chemical exposures and hair dye use. The validity and reproducibility of the FFQ have been verified previously ${ }^{(10,11)}$, with the intraclass correlation coefficient for mean daily intake of total soya foods being 0.78 in a test-retest ${ }^{(11)}$.

\section{Dietary intake assessment}

Habitual food intakes were assessed using the quantitative FFQ. A 'reference' recall period was set as 1 year before the diagnosis in cases and 1 year before the date of interview for controls. If there were recent alterations in eating habits, information was sought on the habits before the change. The frequency of food intakes was classified into nine categories: never or hardly ever, once a month, two to three times a month, once a week, two to three times a week, four to six times a week, once a day, twice a day and $\geq 3$ times a day. The usual amount of each food consumed per meal was estimated using the common Chinese unit liang ( 1 liang $=50 \mathrm{~g}$ ). The frequency and quantity variables derived from the FFQ were converted into daily intake, taking seasonal factors and market availability into account. In line with previous studies ${ }^{(12,13)}$, soya foods included nine items of soya and soya-based foods (dry soyabean, fresh green soyabean, soyabean milk, skin of soyabean milk, fresh bean curd, textured bean curd, fried bean curd, fermented bean curd and soyabean sprouts). Daily intakes (mg) of daidzein, genistein, glycitein and total isoflavones were calculated using an updated version of the US Department of Agriculture Isoflavone Database $^{(14)}$, as the contents of isoflavones in foods were unavailable from the Chinese Food Composition Tables. Daily intake of isoflavones was estimated on the basis of the amounts of nine-item soya foods consumed, as other food sources are very low in isoflavones and their contribution to total intake can be considered negligible.

\section{Statistical analyses}

Intakes of daidzein, genistein, glycitein and total isoflavones were divided into tertiles based on the distribution of each isoflavone variable in controls. Total energy intake derived from 107 foods was estimated using the 2009 China Food Composition Tables, accounting for seasonal factors and edible portions of foods ${ }^{(15)}$. Other variables were defined as follows: BMI was calculated as body weight in kilograms divided by the square of height in metres $\left(\mathrm{kg} / \mathrm{m}^{2}\right)$; metabolic equivalent task hours per week during the past year was a measure of physical activity; cigarette smoking was defined as a total consumption of twenty packs of cigarettes or more in lifetime, and the cumulative quantity of smoking exposure was indicated as pack-year (number of packs cigarettes consumed per $\mathrm{d}$ multiplied by number of years smoking); ever consumed liquor, beer, wine or any combination was classified as alcohol consumption; drinking tea $\geq 1$ time/month was defined as tea consumption; ever used permanent hair dye in a lifetime was defined as hair dye use; participants self-reporting lifetime exposure to chemicals, either on a job or a hobby, at least $8 \mathrm{~h} /$ week for 1 year or more was defined as ever exposed ${ }^{(16)}$.

Sample characteristics, soya foods and dietary intake of isoflavones were compared between cases and controls. OR and $95 \%$ CI were calculated from conditional logistic regression models ${ }^{(17)}$ to estimate the risk of MDS according to tertiles of isoflavone intake. The lowest intake tertile served as the reference category in all models. In addition to control by design for matching factors, the multivariate analyses were adjusted for 
education (none, primary, secondary, tertiary), BMI $\left(\mathrm{kg} / \mathrm{m}^{2}\right.$, continuous), cigarette smoking (pack-years, continuous), tea consumption (no, yes), alcohol consumption (abstainers, everdrinkers) and daily intakes of energy $(\mathrm{kJ}(\mathrm{kcal}))$, vegetables $(\mathrm{g})$, fruits $(\mathrm{g})$ and red meat $(\mathrm{g})$. These variables were included in regression models because they were associated with MDS risk based on univariate analyses or previous study ${ }^{(18)}$, and associated with soya food intake as a priori confounders ${ }^{(19)}$. Other possible risk factors for MDS, such as chemical exposure and hair dye use, appeared to be disassociated with soya food intake and did not materially alter the results after adjustment; thus, they were excluded from the final model. Variables representing isoflavone intake were subjected to tests for trend by likelihood ratio tests using the original values in continuous format. All $P$ values were based on likelihood ratio statistics and were considered statistically significant if $<0 \cdot 05$. The analyses were conducted with SAS version 9.3 (SAS Institute Inc.).

If the difference in the mean dietary isoflavone intake of matched pairs is 4 (sD 20), a total of 198 case-control pairs were needed with a type I error rate of 0.05 and $80 \%$ power $^{(20)}$. Thus, the sample size of this study provided adequate power to detect differences in dietary isoflavone intake between cases and controls.

\section{Results}

The selected characteristics of MDS cases and controls are presented in Table 1. There was no significant difference between cases and controls on matching factors, BMI, physical activity, mean daily intakes of energy, vegetables and red meat, cancer history in first-degree relatives and hair dye use. Compared with controls, cases attained less education. Cases were more likely than controls to have smoked, abstained from alcohol and to have been ever exposed to chemicals. Cases tended to consume less tea and fruits than controls.

Table 2 describes soya food consumption and dietary intake of isoflavones by case-control status. The vast majority of soya foods consumed in cases and controls were non-fermented products such as soyabean, soyabean milk, fresh bean curd and textured bean curd. Compared with the controls, MDS cases had lower mean daily intakes of most individual soya foods, although most differences did not reach statistical significance. With respect to isoflavones, daily intake of total isoflavones was significantly lower in cases (mean 19.0 (SE 1.4)) than in controls (mean 23.0 (sE 1.3)). Significantly lower intakes of daidzein and genistein were observed in cases when compared with those in controls.

Among cases, median daily intake of total isoflavones ranged from $5.1 \mathrm{mg}$ in the lowest tertile to $36.8 \mathrm{mg}$ in the highest tertile, which was less than that in controls at 7.8 and $41.0 \mathrm{mg}$, respectively. Table 3 shows the associations between dietary isoflavone intake and MDS risk. Higher dietary intake of isoflavones was associated with a decreased risk of MDS. The adjusted OR (95\% CI) for MDS in the highest tertile of intake compared with the lowest tertile were 0.43 (95\% CI $0.21,0.85$ ) for daidzein, 0.36 (95\% CI $0 \cdot 18,0.74)$ for genistein, $0.49(95 \%$ CI $0 \cdot 25,0.97)$ for glycitein and $0.40(95 \%$ CI $0 \cdot 20,0 \cdot 81)$ for total isoflavones. Inverse dose-response trends were apparent for the intakes of daidzein, genistein and total isoflavones ( $P_{\text {for }}$ trend $=0.04$ ), and a marginally significant trend was observed for the intake of glycitein $\left(P_{\text {for trend }}=0 \cdot 10\right)$.

\section{Discussion}

The present case-control study conducted in a Chinese population, whose diet is rich in soya, is the first epidemiological study to date that has examined the association between dietary isoflavone intake and MDS risk. A consistent and statistically significant reduced risk of MDS was observed with high dietary intakes of total isoflavones, and the subclasses of daidzein and genistein.

Many studies have focused on the anti-oestrogenic properties of isoflavones for preventing hormone-related cancers, such as ovarian, breast and prostate cancers ${ }^{(6)}$. Isoflavones may also have a role in the prevention of non-hormone-mediated cancers, as they act as antioxidants and possess anti-carcinogenic properties, such as inhibiting cell growth and angiogenesis, inhibiting both protein tyrosine kinase and DNA topoisomerase II and inducing differentiation ${ }^{(6,21,22)}$. Genistein, the key isoflavone present in soya foods, has exhibited anti-leukaemic activity in a number of in vivo and in vitro studies. For instance, genistein may induce the differentiation of human promyelocytic HL-60 leukaemia cells ${ }^{(23)}$. One study has suggested that genistein not only produces dose- and time-dependent antineoplastic activity against myeloid leukaemic cell lines but also reactivates tumour suppressor genes silenced by aberrant DNA methylation $^{(24)}$. Furthermore, researchers have observed a significant anti-leukaemic effect in mice induced by a genisteinenriched diet, the concentrations of which are observable in the plasma of high soya consumers ${ }^{(24)}$. Although no study is available on the biological effects of isoflavones on MDS, the inferences of the laboratory work are plausible given that MDS shares several characteristics with leukaemia and is characterised by frequent (approximately $30 \%$ ) progression to acute myeloid leukaemia ${ }^{(25)}$. The findings from experimental studies on leukaemia potentially provide an underlying biological explanation for our observation that high dietary intake of isoflavones is associated with a reduced risk of MDS in a population with a soya-rich diet.

The strengths and limitations of the study should be considered. A major strength of the study was that extensive information on soya food intake, as well as other dietary factors, was sought from face-to-face interviews using a validated and reliable questionnaire specifically developed for adult Chinese $^{(10,11)}$. The FFQ used in the study included nine-item soya foods, which were commonly consumed in China and were the major sources of dietary isoflavones. The average daily intake of total isoflavones in the controls was comparable to that in our previous study $(23.0 v .19 .5 \mathrm{mg} / \mathrm{d})^{(12)}$. Moreover, the bioavailability of self-reported usual intake of soya isoflavones has been assessed in previous literature, from which there were strong correlations between plasma concentrations, urinary excretion of isoflavones and self-reported soya intake measured by $\operatorname{FFQ}^{(26,27)}$, suggesting that the participants' self-reported 
Table 1. Selected characteristics between myelodysplastic syndromes cases and controls (Mean values with their standard errors; number of cases and controls and percentages)

\begin{tabular}{|c|c|c|c|c|c|}
\hline \multirow[b]{2}{*}{ Characteristics } & \multicolumn{2}{|c|}{ Cases ( $n$ 208) } & \multicolumn{2}{|c|}{ Controls ( $n$ 208) } & \multirow[b]{2}{*}{$P^{*}$} \\
\hline & $n$ & $\%$ & $n$ & $\%$ & \\
\hline \multicolumn{6}{|l|}{ Age at interview (years) } \\
\hline Mean & \multirow{2}{*}{\multicolumn{2}{|c|}{$\begin{array}{c}54.8 \\
1.1\end{array}$}} & \multicolumn{2}{|c|}{54.5} & \\
\hline SE & & & & & \\
\hline $19-29$ & 16 & $7 \cdot 7$ & 16 & $7 \cdot 7$ & \\
\hline 30-39 & 17 & $8 \cdot 2$ & 17 & $8 \cdot 2$ & \\
\hline $40-49$ & 39 & $18 \cdot 8$ & 39 & $18 \cdot 8$ & \\
\hline $50-59$ & 56 & $26 \cdot 9$ & 56 & $26 \cdot 9$ & \\
\hline $60-69$ & 40 & $19 \cdot 2$ & 40 & $19 \cdot 2$ & \\
\hline $70-86$ & 40 & $19 \cdot 2$ & 40 & $19 \cdot 2$ & - \\
\hline Male & 110 & $52 \cdot 9$ & 110 & $52 \cdot 9$ & - \\
\hline \multicolumn{6}{|l|}{ Residential locality } \\
\hline Urban & 120 & $57 \cdot 7$ & 120 & 57.7 & \\
\hline Rural & 88 & $42 \cdot 3$ & 88 & $42 \cdot 3$ & - \\
\hline \multicolumn{6}{|l|}{ Education } \\
\hline None & 25 & $12 \cdot 0$ & 12 & $5 \cdot 8$ & \\
\hline Primary & 64 & $30 \cdot 8$ & 45 & $21 \cdot 6$ & \\
\hline Secondary & 88 & $42 \cdot 3$ & 58 & $27 \cdot 9$ & \\
\hline Tertiary & 31 & 14.9 & 93 & $44 \cdot 7$ & $<0.001$ \\
\hline \multicolumn{6}{|l|}{ BMI $\left(\mathrm{kg} / \mathrm{m}^{2}\right)$} \\
\hline Mean & \multicolumn{2}{|c|}{$\begin{array}{c}22.9 \\
0.2\end{array}$} & \multicolumn{2}{|c|}{$22 \cdot 9$} & \\
\hline SE & \multicolumn{2}{|c|}{0.2} & \multirow{2}{*}{\multicolumn{2}{|c|}{0.2}} & 0.94 \\
\hline \multicolumn{5}{|l|}{ Physical activity (MET h/week) } & \\
\hline Low $(<38.8)$ & 62 & 29.8 & 69 & $33 \cdot 2$ & \\
\hline Medium $(38 \cdot 8-87 \cdot 6)$ & 54 & $26 \cdot 0$ & 70 & 33.7 & \\
\hline High $(>87.6)$ & 92 & $44 \cdot 2$ & 69 & $33 \cdot 2$ & 0.08 \\
\hline Cigarette smoking & 78 & 37.5 & 60 & $28 \cdot 8$ & 0.01 \\
\hline Alcohol consumption & 76 & 36.5 & 101 & $48 \cdot 6$ & 0.002 \\
\hline Tea consumption & 89 & $42 \cdot 8$ & 140 & $67 \cdot 3$ & $<0.001$ \\
\hline \multicolumn{6}{|l|}{ Energy intake $(\mathrm{kJ} / \mathrm{d})$} \\
\hline Mean & \multicolumn{2}{|c|}{$9851 \cdot 6$} & \multicolumn{2}{|c|}{9698.9} & \\
\hline SE & \multirow{2}{*}{\multicolumn{2}{|c|}{$237 \cdot 2$}} & \multicolumn{2}{|c|}{234.7} & 0.59 \\
\hline \multicolumn{4}{|l|}{ Energy intake (kcal/d) } & & \\
\hline Mean & \multicolumn{2}{|c|}{2354.6} & & & \\
\hline SE & & & & & 0.59 \\
\hline Vegetable intake $(\mathrm{g} / \mathrm{d})$ & & & & & \\
\hline Mean & & & & & \\
\hline SE & & & & & 0.50 \\
\hline Fruit intake (g/d) & & & & & \\
\hline Mean & & & & & \\
\hline SE & & & & & 0.01 \\
\hline Red meat intake $(\mathrm{g} / \mathrm{d})$ & & & & & \\
\hline Mean & & & & & \\
\hline SE & & & & & 0.69 \\
\hline Cancer history in first-degree relatives & 39 & $18 \cdot 8$ & 44 & $21 \cdot 2$ & 0.54 \\
\hline Lifetime exposed to chemicals & 52 & $25 \cdot 0$ & 18 & $8 \cdot 7$ & $<0.001$ \\
\hline Hair dye use & 100 & $48 \cdot 1$ & 99 & $47 \cdot 6$ & 0.92 \\
\hline
\end{tabular}

MET, metabolic equivalent task.

* $P$ value from univariate conditional logistic regression with $1 \mathrm{df}$

usual soya intake derived from the FFQ should have provided a reliable measure of isoflavone intake. Another characteristic was that the multivariate conditional logistic regression analyses were adjusted for a variety of dietary and non-dietary factors, such as the intakes of vegetables, fruits, red meat, energy and cumulative exposure to cigarette smoking. Thus, residual confounding seems unlikely to have had a substantial impact on the risk estimates. Although the univariate analyses show that lifetime exposure to chemicals was significantly associated with MDS risk, it was not significantly associated with dietary isoflavone intake in the control series. Thus, there is no a priori or empirical justification for viewing chemical exposures as a potential confounder in this analysis; if we nevertheless proceed to 'over-adjust' by inclusion of chemical exposures as a covariate, the price paid is an unwarranted loss of precision in estimates concerning our primary research question.

The study is thought to have introduced little selection bias. The cases were identified from medical records at the participating hospital. Relevant medical records were reviewed daily and eligible patients diagnosed in this MDS Centre during the study period were invited to participate in the study with a $95 \%$ response proportion. Thus, the systematic identification procedure ensured that the ascertainment of cases was maximised and near complete. The controls selected from outpatient 
Table 2. Soya food consumption and dietary intake of isoflavones between myelodysplastic syndromes cases and controls (Mean values with their standard errors)

\begin{tabular}{|c|c|c|c|c|c|}
\hline \multirow[b]{2}{*}{ Daily intake } & \multicolumn{2}{|c|}{ Cases (n 208) } & \multicolumn{2}{|c|}{ Controls ( $n$ 208) } & \multirow[b]{2}{*}{$P^{\star}$} \\
\hline & Mean & SE & Mean & $\mathrm{SE}$ & \\
\hline \multicolumn{6}{|l|}{ Soya foods (g or ml) } \\
\hline Dry soyabean & $2 \cdot 0$ & 0.7 & 3.6 & 0.7 & 0.10 \\
\hline Fresh green soyabean & 7.9 & 0.9 & 6.5 & 0.5 & 0.16 \\
\hline Soyabean milk & 33.1 & $5 \cdot 8$ & 33.9 & $4 \cdot 1$ & 0.90 \\
\hline Skin of soyabean milk & $2 \cdot 7$ & 0.4 & $3 \cdot 7$ & 0.6 & 0.13 \\
\hline Fresh bean curd & 24.9 & $2 \cdot 3$ & $24 \cdot 7$ & $2 \cdot 0$ & 0.95 \\
\hline Textured bean curd & $10 \cdot 6$ & 1.6 & 13.8 & 1.5 & 0.14 \\
\hline Fried bean curd & $2 \cdot 4$ & 0.3 & 3.8 & 0.7 & 0.04 \\
\hline Fermented bean curd & 0.7 & 0.2 & 0.7 & $0 \cdot 1$ & 0.96 \\
\hline Soyabean sprouts & $3 \cdot 2$ & 0.5 & $6 \cdot 6$ & 0.9 & 0.001 \\
\hline \multicolumn{6}{|l|}{ Isoflavones (mg) } \\
\hline Daidzein & 8.0 & 0.6 & 9.7 & 0.6 & 0.04 \\
\hline Genistein & $10 \cdot 0$ & 0.7 & $12 \cdot 1$ & 0.7 & 0.04 \\
\hline Glycitein & 1.8 & 0.1 & $2 \cdot 1$ & 0.1 & 0.12 \\
\hline Total isoflavones & $19 \cdot 0$ & 1.4 & $23 \cdot 0$ & 1.3 & 0.04 \\
\hline
\end{tabular}

Table 3. Associations between dietary intake of isoflavones and myelodysplastic syndromes risk (Odds ratios and $95 \%$ confidence intervals)

\begin{tabular}{|c|c|c|c|c|c|c|c|c|}
\hline \multirow[b]{2}{*}{ Daily intake* } & \multirow[b]{2}{*}{ Cases (n 208) } & \multirow[b]{2}{*}{ Controls ( $n$ 208) } & \multicolumn{3}{|c|}{ Univariate model† } & \multicolumn{3}{|c|}{ Multivariate model } \\
\hline & & & OR & $95 \% \mathrm{Cl}$ & $P_{\text {for trend }} \S$ & OR & $95 \% \mathrm{Cl}$ & $P_{\text {for trend }} \S$ \\
\hline Daidzein (mg) & & & & & 0.04 & & & 0.04 \\
\hline $3.2(0.0$ to $<4.8)$ & 95 & 69 & 1.00 (Ref.) & & & 1.00 (Ref.) & & \\
\hline $7.2(4.8$ to $<10.4)$ & 62 & 70 & 0.67 & $0.43,1.04$ & & 0.72 & $0.40,1.31$ & \\
\hline $17.3(10.4$ to 51.4$)$ & 51 & 69 & 0.54 & $0.34,0.88$ & & 0.43 & $0.21,0.85$ & \\
\hline Genistein (mg) & & & & & 0.04 & & & 0.04 \\
\hline $3.8(0.0$ to $<6.0)$ & 97 & 68 & 1.00 (Ref.) & & & 1.00 (Ref.) & & \\
\hline $8.8(6.0$ to $<13.5)$ & 57 & 71 & 0.57 & $0.36,0.91$ & & 0.49 & $0.26,0.94$ & \\
\hline $21.5(13.5$ to 57.3$)$ & 54 & 69 & 0.55 & $0.34,0.89$ & & 0.36 & $0.18,0.74$ & \\
\hline Glycitein (mg) & & & & & $0 \cdot 12$ & & & $0 \cdot 10$ \\
\hline $0.7(0.0$ to $<1.1)$ & 96 & 69 & 1.00 (Ref.) & & & 1.00 (Ref.) & & \\
\hline $1.7(1.1$ to $<2.2)$ & 53 & 69 & 0.57 & $0.36,0.91$ & & 0.38 & $0.19,0.74$ & \\
\hline $3.4(2 \cdot 2$ to $10 \cdot 3)$ & 59 & 70 & 0.62 & $0.38,0.98$ & & 0.49 & $0.25,0.97$ & \\
\hline Total isoflavones (mg) & & & & & 0.04 & & & 0.04 \\
\hline $7.8(0.0$ to $<11.5)$ & 94 & 69 & 1.00 (Ref.) & & & 1.00 (Ref.) & & \\
\hline $16.9(11.5$ to $<25.7)$ & 64 & 70 & 0.69 & $0.44,1.07$ & & 0.64 & $0.35,1.19$ & \\
\hline $41.0(25.7$ to 116.4$)$ & 50 & 69 & 0.53 & $0.33,0.87$ & & 0.40 & $0.20,0.81$ & \\
\hline
\end{tabular}

Ref., referent values.

* Median and range of each tertile intake in controls.

† Estimates from separate univariate conditional logistic regression model with sex, birth quinquennium and residential locality (urban or rural) as matching variables.

‡ Estimates from separate conditional logistic regression adjusted for education (none, primary, secondary, tertiary), BMI (kg/m², continuous), cigarette smoking (pack-years, continuous), alcohol consumption (abstainers, ever-drinkers), tea consumption (no, yes), intakes of energy (kJ (kcal)/d, continuous), vegetables (g/d, continuous), fruits (g/d, continuous) and red meat ( $\mathrm{g} / \mathrm{d}$, continuous).

$\S$ Likelihood ratio tests for trend using the original values in continuous format.

departments of the same hospital were considered as a valid study base sample, as evidenced by the remarkably similar distributions of dietary isoflavone intake, as well as demographic characteristics, lifestyle factors between hospital outpatient controls and community controls in China ${ }^{(13,28,29)}$.

With regard to the likelihood of information bias, the potential protective role of soya foods against MDS had not received attention before nor at the time of interview, and thus information bias from that source was unlikely. Dietary habits may change because of disease status in cases. Nevertheless, it appears unlikely that disease status materially affected self-reported responses, as participants' usual dietary intake was measured using a 'reference' recall period. If non-differential misclassification of dietary intake occurred, the resultant bias would have been towards the null and could not have accounted for the inverse association reported here. We made an effort to minimise recall bias by asking study participants to report their usual dietary intake during the 1 year before the diagnosis in cases and 1 year before the interview date for controls. In this study, $92.8 \%$ of the cases and $99.0 \%$ of the controls reported that their diets in the previous year were typical of their usual diets. Other personal life habits as 
confounding factors that were adjusted for in regression analyses, including cigarette smoking, tea consumption and alcohol consumption, were relatively easier for participants to recall with reasonable accuracy. Last, it was impractical to evaluate the effect of isoflavone intake by sex and by MDS subtype because of the limitations of the study sample size.

In conclusion, the study suggests an inverse association between the intake of dietary isoflavones and MDS risk in a Chinese population. Future studies are needed to confirm or refute these results and to characterise the underlying biological mechanisms, which may have longer-term therapeutic potential.

\section{Acknowledgements}

The authors are grateful to the participants of this study. The authors thank Hong-Yan Tong, Li-Hong Cao and other staff from The First Affiliated Hospital of Zhejiang University for their kind assistance with fieldwork. P. L. was supported by the Scholarship for International Research Fees and the University Postgraduate Award of The University of Western Australia.

This research received no specific grant from any funding agency, commercial or not-for-profit sectors.

The authors' contributions are as follows: P. L., C. D'A. J. H., J. J. and M. Z. designed the research project; P. L., J. J. and M. Z. managed the data; P. L. carried out the statistical analyses; P. L. wrote the article; C. D'A. J. H. and M. Z. critically revised the article; P. L., C. D'A. J. H. and M. Z had primary responsibility for the final content.

There are no conflicts of interest to declare.

\section{References}

1. Tefferi A \& Vardiman JW (2009) Myelodysplastic syndromes. $N$ Engl J Med 361, 1872-1885.

2. Wang W, Wang H, Wang XQ, et al. (2012) First report of incidence of adult myelodysplastic syndrome in China. Ann Hematol 91, 1321-1322.

3. Chihara D, Ito H, Katanoda K, et al. (2014) Incidence of myelodysplastic syndrome in Japan. J Epidemiol 24, 469-473.

4. Rollison DE, Howlader N, Smith MT, et al. (2008) Epidemiology of myelodysplastic syndromes and chronic myeloproliferative disorders in the United States, 2001-2004, using data from the NAACCR and SEER programs. Blood 112, 45-52.

5. Rodger EJ \& Morison IM (2012) Myelodysplastic syndrome in New Zealand and Australia. Intern Med J 42, 1235-1242.

6. Messina MJ, Persky V, Setchell KD, et al. (1994) Soy intake and cancer risk: a review of the in vitro and in vivo data. Nutr Cancer 21, 113-131.

7. Murphy PA, Barua K \& Hauck CC (2002) Solvent extraction selection in the determination of isoflavones in soy foods. J Chromatogr B Analyt Technol Biomed Life Sci 777, 129-138.

8. Tse G \& Eslick GD (2014) Soy and isoflavone consumption and risk of gastrointestinal cancer: a systematic review and meta-analysis. Eur J Nutr (Epublication ahead of print version 30 December 2014)

9. Liu P, Zhang M, Jin J, et al. (2015) Tea consumption reduces the risk of de novo myelodysplastic syndromes. Leuk Res 39 , 164-169.
10. Shu XO, Yang G, Jin F, et al. (2004) Validity and reproducibility of the food frequency questionnaire used in the Shanghai Women's Health Study. Eur J Clin Nutr 58, 17-23.

11. Zhang M, Binns CW \& Lee AH (2005) A quantitative food frequency questionnaire for women in southeast China: development and reproducibility. Asia Pac J Public Health 17, 29-35.

12. Zhang M, Yang H \& Holman CD (2009) Dietary intake of isoflavones and breast cancer risk by estrogen and progesterone receptor status. Breast Cancer Res Treat 118, 553-563.

13. Li L, Zhang M \& Holman CD (2013) Population versus hospital controls in the assessment of dietary intake of isoflavone for case-control studies on cancers in China. Nutr Cancer 65 , 390-397.

14. US Department of Agriculture, Agricultural Research Service (2008) USDA database for the isoflavone content of selected foods, release 2.0. http://www.ars.usda.gov/nutrientdata/isoflav (accessed August 2014).

15. National Institute of Nutrition and Food Safety, Chinese Centre for Disease Control and Prevention (2009) China Food Composition, 2nd ed., vol. 1. Beijing: Peking University Medical Press.

16. Strom SS, Gu Y, Gruschkus SK, et al. (2005) Risk factors of myelodysplastic syndromes: a case-control study. Leukemia 19, 1912-1918.

17. Breslow NE \& Day NE (1980) Statistical Method in Cancer Research: Vol. 1 - The Analysis of Case-Control Studies, IARC Scientific Publications No. 32. Lyon: IARC.

18. Ma X, Lim U, Park Y, et al. (2009) Obesity, lifestyle factors, and risk of myelodysplastic syndromes in a large US cohort. Am J Epidemiol 169, 1492-1499.

19. Miettinen OS \& Cook EF (1981) Confounding: essence and detection. Am J Epidemiol 114, 593-603.

20. Dupont WD \& Plummer WD Jr (1990) Power and sample size calculations. A review and computer program. Control Clin Trials 11, 116-128.

21. Fotsis T, Pepper M, Adlercreutz H, et al. (1995) Genistein, a dietary ingested isoflavonoid, inhibits cell proliferation and in vitro angiogenesis. J Nutr 125, 790S-797S.

22. Markovits J, Linassier C, Fosse P, et al. (1989) Inhibitory effects of the tyrosine kinase inhibitor genistein on mammalian DNA topoisomerase II. Cancer Res 49, 5111-5117.

23. Constantinou A, Kiguchi K \& Huberman E (1990) Induction of differentiation and DNA strand breakage in human HL-60 and K-562 leukemia cells by genistein. Cancer Res 50, 2618-2624.

24. Raynal NJ, Momparler L, Charbonneau M, et al. (2008) Antileukemic activity of genistein, a major isoflavone present in soy products. J Nat Prod 71, 3-7.

25. Corey SJ, Minden MD, Barber DL, et al. (2007) Myelodysplastic syndromes: the complexity of stem-cell diseases. Nat Rev Cancer 7, 118-129.

26. Wu AH, Yu MC, Tseng CC, et al. (2004) Plasma isoflavone levels versus self-reported soy isoflavone levels in AsianAmerican women in Los Angeles county. Carcinogenesis 25 , $77-81$.

27. Maskarinec G, Singh S, Meng L, et al. (1998) Dietary soy intake and urinary isoflavone excretion among women from a multiethnic population. Cancer Epidemiol Biomarkers Prev $\mathbf{7}$, 613-619.

28. Li L, Zhang M \& Holman D (2011) Population versus hospital controls for case-control studies on cancers in Chinese hospitals. BMC Med Res Methodol 11, 167.

29. Li L, Zhang M \& Holman CD (2013) Hospital outpatients are satisfactory for case-control studies on cancer and diet in China: a comparison of population versus hospital controls. Asian Pac J Cancer Prev 14, 2723-2729. 